\title{
Expanding one-pot cell-free protein synthesis and immobilization for on- demand manufacturing of biomaterials
}

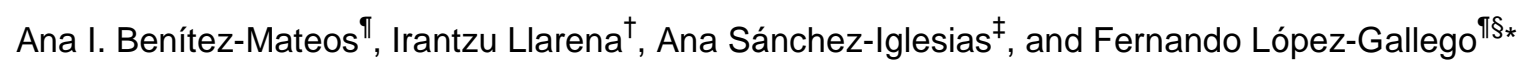

"Heterogeneous Biocatalysis Group. CIC biomaGUNE. Paseo Miramón 182. Edificio empresarial "C". 20014. San Sebastián (Spain)

†Optical Spectroscopy Platform. CIC biomaGUNE. Paseo Miramón 182. Edificio empresarial "C". 20014. San Sebastián (Spain)

${ }^{\ddagger}$ Colloidal Nanofabrication Platform. CIC biomaGUNE. Paseo Miramón 182. Edificio empresarial "C". 20014. San Sebastián (Spain)

${ }^{\S}$ ARAID, Aragon I+D foundation, Zaragoza, (Spain)

* flopezgallego@unizar.es

\section{ABSTRACT}

Fabrication of protein-based biomaterials is an arduous and time-consuming procedure with multiple steps. In this work, we describe a portable tool-kit that integrates both cell-free protein synthesis (CFPS) and protein immobilization in one-pot, just by mixing DNA, solid materials and a CFPS system. We construct a modular set of plasmids that fuse the N-terminus of superfolded Green Fluorescent Protein (sGFP) with different peptide-tags (poly-(6X)Cys, poly-(6X)His and poly-(6X)Lys), which drive the immobilization of the protein on the tailored material (agarose beads with different functionalities, gold nanorods and silica nanoparticles). This system also enables the incorporation of azide-based amino acids into the nascent protein for its selective immoblization through copper-free click reactions. Finally, this technology has been expanded to the synthesis and immobilization of enzymes and antibody-binding proteins for the fabrication of functional biomaterials. This synthetic biological platform emerges as a versatile tool for the on-demand fabrication of therapeutic, diagnostic and sensing biomaterials. 


\section{TABLE OF CONTENTS}

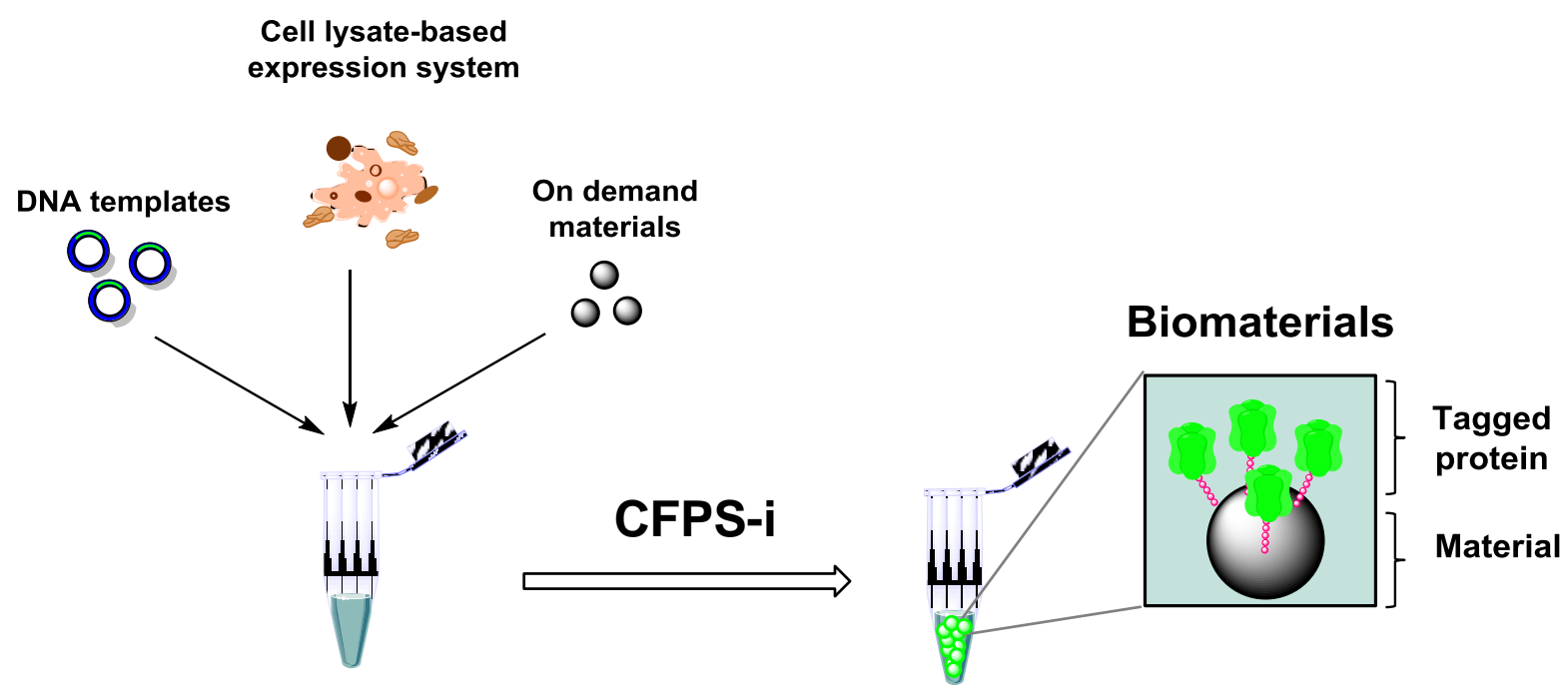

\section{KEYWORDS}

Cell-free protein synthesis, site-selective protein immobilization, biomaterials, synthetic biology, pointof-care 


\section{INTRODUCTION}

The utilization of biomaterials for biomedical purposes is exponentially growing in the last decade. ${ }^{1}$ Consequently, a large variety of biomaterials functionalized with proteins are emerging as therapeutic agents $^{2}$ diagnostic devices ${ }^{3}$, biosensors ${ }^{4}$ and contrast agents for molecular imaging. ${ }^{5}$ In terms of biological activity, proteins (antibodies, cytokines receptors, ligands, enzymes, etc.) are among the most interesting biomolecules to functionalize the solid materials. ${ }^{6}$ Generally, the manufacturing of protein-based biomaterials includes: 1) synthesis of suitable materials for certain application, 2) expression and purification of the protein and 3) immobilization of the protein on the solid material. ${ }^{7}$ This multi-step workflow is designed for bulk production of biomaterials in sophisticated and centralized production facilities, but it fails when the manufactured products need to be deployed at the point-of-care or are economically non-viable at small scales like orphan drugs. ${ }^{8}$

The major restriction for rapid and efficient protein-based biomaterial manufacturing relies on the stability of the biological element. The production and storage of proteins is tied to its labile nature, which limits their delivery to remote areas where the cold chain cannot be assured. Another hurdle for the rapid manufacturing of this type of biomaterials is the biosafety regulations when proteins are produced in living organisms, which require specific skills and specialized facilities, limiting the operation in low-resource areas. Finally, once the protein is conjugated to the material, the greatest challenge is preserving the biological function of the biomaterials during storage and distribution.

On-demand synthesis of protein-functionalized biomaterials at the point of care requires the rapid synthesis and immobilization of the protein in one-pot. However, the multi-pot in vivo protein synthesis and purification are time-consuming and hardly miniaturizable. Alternatively, cell-free protein synthesis (CFPS) is a promising technology to readily synthesize well-folded proteins ${ }^{9}$ on-demand since crude extract have been successfully engineered to maximize the productivity and sustain the energy levels that assures competitive protein yields compared to in vivo systems. ${ }^{10,11}$ Hence, incorporating CFPS to biomaterial manufacturing allows the simultaneous protein synthesis and immobilization in the same test tube. Now, the fabrication system only relies on the stability of the materials and the freeze-dried cell-free extract that have demonstrated their longevity under standard storage conditions. ${ }^{12-14}$ 
To this aim, the immobilization chemistry must be orthogonal to the protein synthesis, avoid unspecific interactions between the protein synthesis machinery and the solid material, and correctly orient the protein to guarantee the functionality of the resulting biomaterial. A previous work showed a CFPS-i of membrane proteins tagged with GFP at their C-terminus and immobilized on PVDF membranes through hydrophobic interactions. ${ }^{15}$ Unfortunately, this kind of hydrophobic interaction is not very selective to expand this technique for new applications. Protein tagging with polypeptide or protein domains is the most widespread strategy to purify proteins for biochemical and structural studies. Furthermore, the tag serves to control the orientation of protein when immobilized on solid materials. ${ }^{16,17}$ Nowadays we have access to a large variety of peptide tags and domains that selectively bind silver, ${ }^{18}$ gold, ${ }^{19}$ nickel oxide ${ }^{20}$ and iron oxide ${ }^{21}$ through coordination and covalent bonds, silica particles ${ }^{22}$ through ionic interactions, biopolymeric particles such as agarose beads ${ }^{23}$ through multivalent interactions based on hydrogen and Van der Waals bonds, and even oil-droplets ${ }^{24}$ through hydrophobic interactions.

A

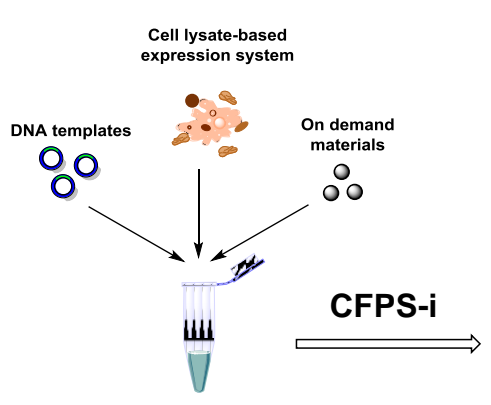

B

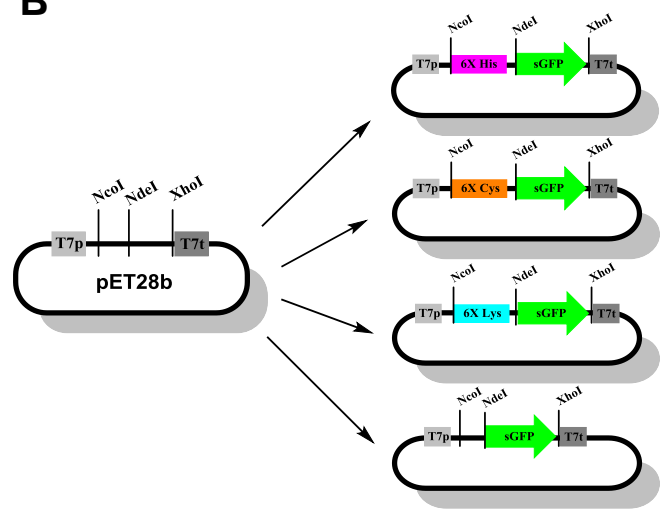

Figure 1. CFPS-i reaction with a set of plasmids encoding sGFP gene. (A) One-pot CFPS-i process for the manufacturing of biomaterials. (B) Modular plasmid set for the biomaterials manufacturing. Plasmid architecture shows the restriction enzymes needed to modularly exchange the target protein and the tag.

In several pioneering works, CFPS was utilized for the fabrication of self-assembling protein microarrays as NAPPA technology, ${ }^{25}$ PISA method ${ }^{26}$ and other protein array-based approaches. ${ }^{15,27,28}$ This concept was further extended to the protein synthesis and selective immobilization by using Histag. ${ }^{29-31}$ These systems have been successfully applied for drug discovery and protein engineering. ${ }^{14,30}$ Likewise, it has been recently reported the synthesis and immobilization of single- 
span membrane proteins on oil drops, as therapeutic biomaterial for killing cultured cancer cells. ${ }^{32}$ This one-pot concept may improve the on-demand fabrication of biomaterials, overall in the prototyping phase of the manufacturing.

Here, we expand the synthetic biological platform coupling cell-free synthesis and immobilization of proteins (CFPS-i) in a single test tube for manufacturing biomaterials (Figure 1A). This portable system may work at the point-of-care just adding DNA templates, CFPS systems and on-demand materials. The toolbox of polypeptide tags and their different immobilization chemistries open a wide range of possibilities for the on-demand fabrication of biomaterials in one-pot. Moreover, the coexpression and co-immobilization of several proteins may improve the scope of this approach. Finally, we extend this technological platform to the production of biomaterials based on enzymes and antibodies demonstrating the biological functionality of the synthesized and immobilized proteins.

\section{RESULTS AND DISCUSSION}

\section{Construction of a plasmid set for CFPS-i: a versatile and modular genetic system}

We fabricated a set of plasmids that enconde superfolded green fluorescent protein (sGFP) fused to the N-terminus with different polypeptide tags. In this construction, both the sGFP and the tag sequences can be easily exchanged by others just by enzymatic digestion (Figure 1B and Figure S1A). Hence, the modular genetic toolbox design enables to easily fuse any target protein with any polypeptide tag. To proof the modularity of this architecture, we constructed three plasmids expressing sGFP harboring at its $\mathrm{N}$-terminus poly-(6x)His (His-sGFP), poly-(6x)Lys (Lys-sGFP) or poly-(6x)Cys (Cys-sGFP) peptides that selectiviely immobilize the protein on different solid materials activated with metal chelates, negative charges or disulfide groups, respectively (Figure 1B). After transformation in E.coli, all these plasmids similarly expressed in vivo the sGFP tagged with the different tags (Figure S2) accordingly to previous results that demostrate that sGFP expression remains unaltered regardless the position ( $\mathrm{N}$ - or $\mathrm{C}$-terminus) and the presence of the tag. ${ }^{33}$ Nevertheless, the in vitro expresion of the untagged SGFP was significatly more efficient than the expresion of the tagged variants which presented different expression levels between them (Figure S3). The in vitro expression of Lys-sGFP was lower than His-sGFP, and significantly lower than Cys-sGFP. These data correlate with the amino acids content in the sGFP primary sequence; 20 lysines, 11 Histidines and 2 cysteines, which suggests that the amino acid or tRNAs pools may be the limiting factor to achieve high protein yields with tagged proteins. 


\section{Cell-free synthesis and immobilization of His-sGFP in one-pot}

To demostrate that protein synthesis and immobilization can occur orthogonally and simultaneously, we synthesized His-sGFP with commercially available S30 cell-free extract from $E$. coli in presence of porous agarose particles activated with cobalt-chelates (AG-Co). Expectedly, protein synthesis was not observed when empty pET28b was used as negative control. On the contrary, when the CFPS system was incubated with the plasmid encoding the untagged sGFP and the beads, the fluorescence appears and remaims in the reaction bulk without selectively colonizing the beads (data not shown). Finally, only when the plasmid expressing the His-sGFP and AG-Co were incubated with the cell-free extract, we observed the selective confinament of the fluorescence in the bead surface. Under this conditions, we were able to load up to $2.8 \pm 0.2 \mathrm{mg}$ of protein per gram of carrier after 12 hours (see METHODS section for more information). These experimental evidences demostrate the in vitro synthesis and concurrent selective immobilization of the protein but only when the protein tag/bead pair is properly selected. To better understand the dynamics of this two-step/onepot process, we performed the synthesis and immobilization of His-sGFP immobilized on AG-Co by measuring sGFP fluorescence along the time (Figure 2).

The on-line monitoring of the process reveals that SGFP gene under the control of T7 promoter is transcribed and translated producing a His-tagged nascent protein that is subsequently immobilized on the AG-Co. As we can see from the time-course images, fluorescence is barely accumulated in the bulk during the entire process (Figure 2A, Movie S1). This fact suggests that the immobilization rate is higher than the synthesis rate, converting the latter into the rate-limiting step. Despite CFPS reactions in solution normally reach the maximum protein yields in 2-4 hours, ${ }^{34,35}$ the accumulation of the nascent proteins inside the porous beads continued for more than 12 hours and never reached a plateau (Figure 2A). Furthermore, we can observe that larger beads are more efficient in recruiting the nascent proteins due to their higher surface (Figure 2A). Finally, the on-line monitoring of the CFPS-i process also reveals a uniform distribution of His-sGFP within the microstructure of the beads (Figure 2B). A similar uniform distribution was found in a similar set-up but using agarose beads activated with nickel-chelates. ${ }^{14}$ Once the CFPS-i system was demonstrated to be functional, we re-used the cell extracts by separating the loaded beads and incubated the cell extracts with fresh empty beads, cofactors and reagents to the cell-free solution. Unfortunately, the protein synthesis yield diminished $96.0 \%$ after the second use (Figure S5). Finally, we freeze-dried the cell extracts to improve the portability and robustness of this system according to recent studies. ${ }^{12,13,16}$ 
We pleasantly achieved the same protein synthesis yield after 12-hour reaction with both, freeze-dried and no freeze-dried cell extracts, although the synthesis rate was 2.63 times slower using the freezedried cell extracts than the commercial solution (Figure S6).

A

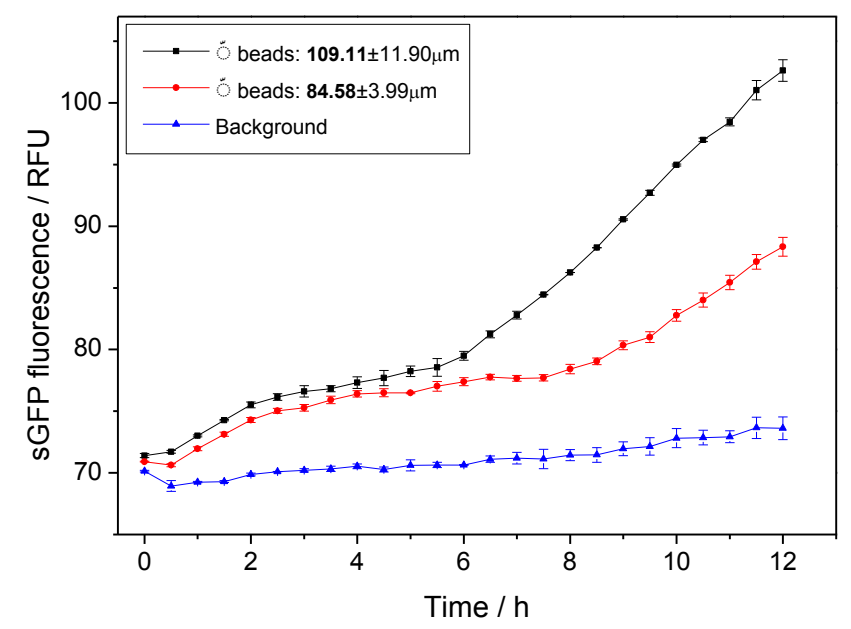

B

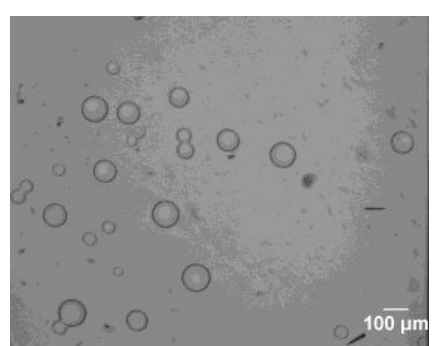

$t=O h$

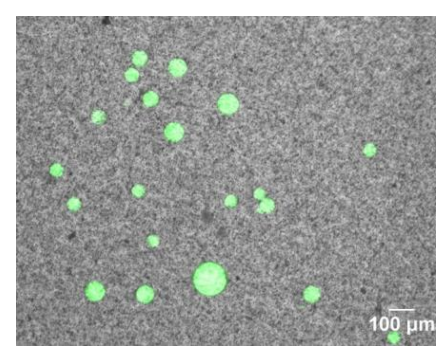

$t=12 h$

Figure 2. On-line monitoring CFPS-i by fluorescence microscopy. (A) sGFP fluorescence inside the beads and in the background (the reaction media outside the beads) along the time. Beads were grouped according to their size. The mean fluorescence value at each time point corresponds to the average fluorescence of 3 beads. (B) Fluorescence microscopy images overlaying brightfield and sGFP signals before (left) and after (right) CFPS-i reaction was completed.

In order to demonstrate that all His-sGFP was immobilized on AG-Co at $2.8 \mathrm{mg} / \mathrm{g}$ of loading, we also performed an immobilization time-course of His-sGFP (in vivo expressed) (Figure S7). Under these conditions, the immobilization was monitor by measuring the sGFP fluorescence inside and outside the AG-Co by using a multi-plate fluorimeter, a method with lower limit of detection. After 12 hours of immobilization reaction, we detected more than $96 \%$ of sGFP on AG-Co, meanwhile less than 
$4 \%$ of the initial solution of in vivo expressed sGFP was detected outside beads. These results sustain a high efficiency of the CFPS-i platform, at least for $2.8 \mathrm{mg} / \mathrm{g}$ of protein loading.

\section{Synthesis on-demand of different biomaterials functionalized with sGFP tagged with different tags}

First of all, we tested the high selectivity of CFPS-i producing Cys-sGFP in presence of two different materials, AG-Co and methacrylate beads activated with epoxide groups (Pu-E). Nascent Cys-sGFP was exclusively immobilized on Pu-E (Figure 3), as result of the specific interaction between the thiol groups of the Cys-tag and the epoxide groups of the carrier.

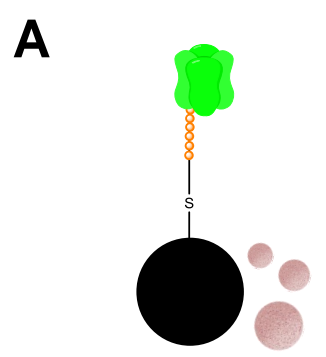

B

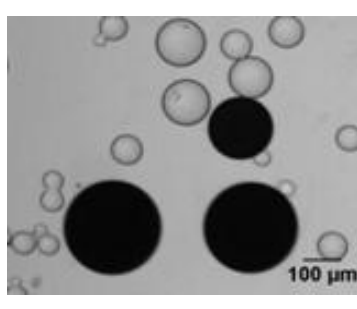

$t=0 h$

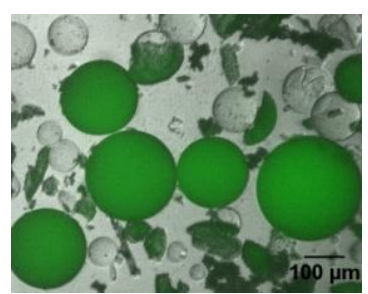

$t=12 h$

Figure 3. CFPS-i of Cys-sGFP in presence of AG-Co and Pu-E. (A) Scheme of the selective binding between the Cys-GFP and Pu-E (black) in presence of AG-Co (pink). (B) Fluorescence microscopy images overlaying brightfield and sGFP signals before and after the CFPS-i reaction was accomplished.

To expand the scope of the CFPS-i methodology, we coupled the synthesis of Cys-sGFP and Lys-sGFP to their selective immobilization on gold nanorods (AuNRs) and silica nanoparticles $\left(\mathrm{SiO}_{2} \mathrm{NPs}\right.$ ), respectively (Figure 4 and Figure S8). Cys-sGFP was efficiently immobilized on the AuNRs due to the formation of thiol-gold covalent bonds (Figure 4A); this chemistry has been extensively exploited in the biofunctionalization of gold-based nanomaterials. ${ }^{36}$ Likewise, Lys-sGFP was selectively and stably absorbed on $\mathrm{SiO}_{2} \mathrm{NPs}$ through the ionic interaction between the positive charges of the $\varepsilon-\mathrm{NH}_{2}$ group of the Lysine-tag and the negatively charged surface of these nanoparticles (Figure 4B), accordingly to the results obtained with other basic domains and tags fused to the protein of interest. ${ }^{22}$ 
A

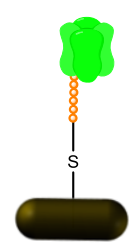

B

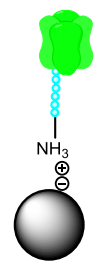

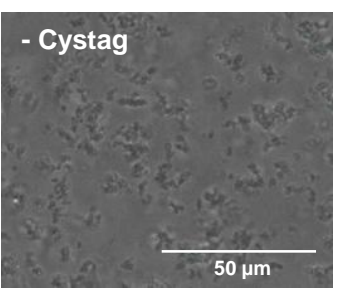
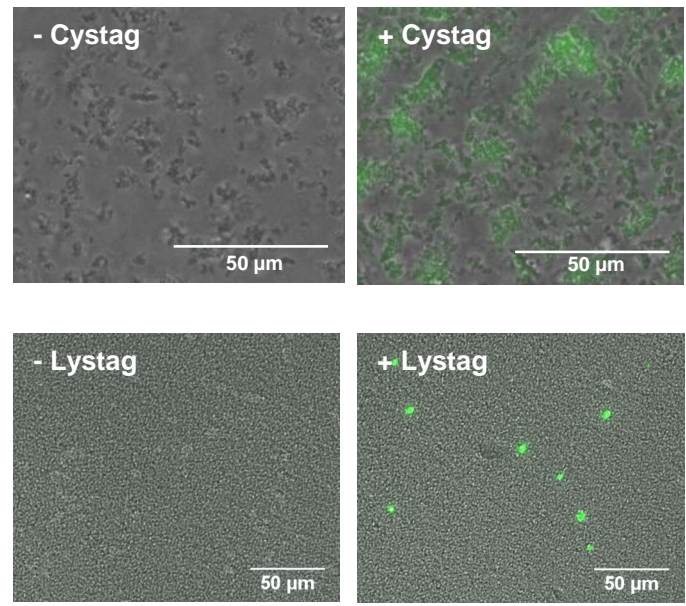

Figure 4. CFPS-i of SGFP onto on-demand materials by selective polypeptide-tags. (A) CyssGFP. (B) Lys-sGFP. From left to right, scheme of the selective binding between the polypeptidetagged sGFP and the nanoparticle, fluorescence microscopy images overlaying brightfield and sGFP signals for the control reaction with untagged SGFP (-Cystag / -Lystag) and for the reaction with polypeptide-tagged sGFP (+Cystag / +Lystag).

In both cases, the untagged sGFP was synthesized but not immobilized on both nanoparticles (Figure 4). The interaction between the Lys-tag and the silica surface is very selective, although we observed some unspecific interactions between proteins from the CFPS machinery and the $\mathrm{SiO}_{2} \mathrm{NPs}$ (Figure S9). In addition, we also demonstrated that free amino acids contained in the $\mathbf{S} 30$ premix plus were not competing with the poly-peptide sGFP for the reactive groups, achieving similar protein immobilization yields with and without free amino acids in the bulk. We observed similar protein immobilization kinetics in both cases (Figure S4).

CFPS-i reaction was also carried out by drop-casting the corresponding plasmid and cell-free protein synthesis machinery on the top of glass slides, letting the reaction mixture react for 12 hours and then intensively washing the glass surface with buffered solution. Herein, the protein synthesis was successfully accomplished since fluorescence was detected in drops containing either tagged or untagged proteins after the reaction time (Figure 5 and Figure S10). After the washing step, the drops containing the plasmid encoding Lys-sGFP displayed fluorescence spots at the glass, while no fluorescence was detected in those drops that in vitro expressed the untagged protein, since it was removed from the surface during the washing step (Figure 5). These experimental data corroborate that the Lys-tag drives the protein immobilization on silica-based materials and expand its utilization to 
other solid architectures. This technology skips the chemical functionalization steps of glass slides required for the conventional proceedings to prepare protein-based microarrays. ${ }^{37}$

A
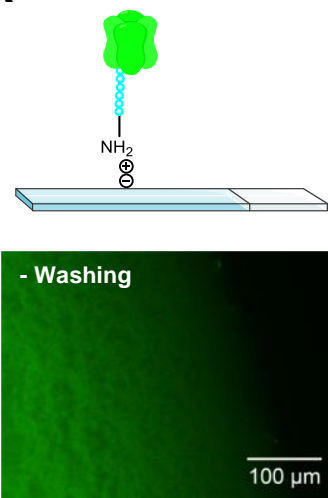

+ Washing
B
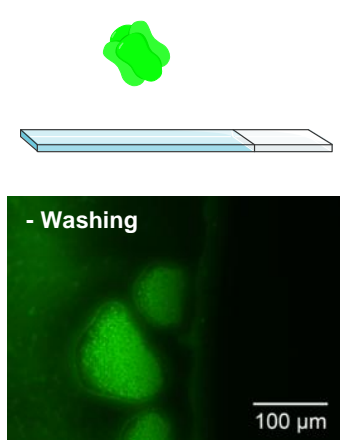

+ Washing

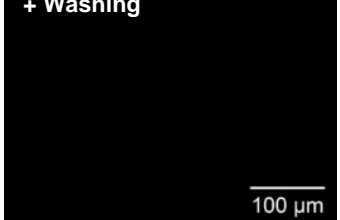

Figure 5. CFPS-i of Lys-sGFP onto glass slides. System expressing (A) Lys-sGFP and (B) untagged SGFP

\section{Incorporation of non-natural amino acids through CFPS to enable bio-orthogonal immobilization mediated by copper-free click chemistry}

In order to go beyond the chemistry offered by the nature, we also expanded the CFPS-i to copper-free click chemistry reactions by incorporating non-natural amino acids harboring azide groups into the nascent proteins and functionalizing materials with cyclooctyne groups. To this aim, we firstly activated agarose beads with cyclooctyne groups through a new surface chemistry protocol starting from commercially available agarose beads activated with cyanogens bromide (Figure S11). Figure 6 shows that protein synthesis fails and fluorescence can be detected neither in the bulk nor inside the beads as expected when neither methionine nor L-AHA (L-azidohomoalanine) is included in the amino acid mixture. Furthermore, when the protein is synthesized with methionine, the protein is produced but immobilization on cyclooctyne-activated agarose beads does not occur, remaining the nascent protein in the reaction bulk. Likewise, when the protein is synthesized with L-AHA but incubated with agarose beads activated with hydroxyl groups instead of cyclooctyne ones, sGFP fluorescence is only detected in the reaction bulk. 
These experimental data demonstrate that the synthesis and copper-free click-immobilization of sGFP is only possible when azide side chains are displayed in the protein surface and the agarose surface is activated with cyclooctyne groups (Figure 6). According to the sGFP X-ray structure (PDB: 2B3P), L-AHA can be introduced at position 1, 78, 88 and 216 of the protein primary sequence. Positions 1, 78 and 88 cluster at the bottom part of the $\beta$-barrel where $\mathrm{N}$ - and $\mathrm{C}$-terminus are located, while position 216 is located at the hollow of the barrel facing the chromophore (Figure S12).

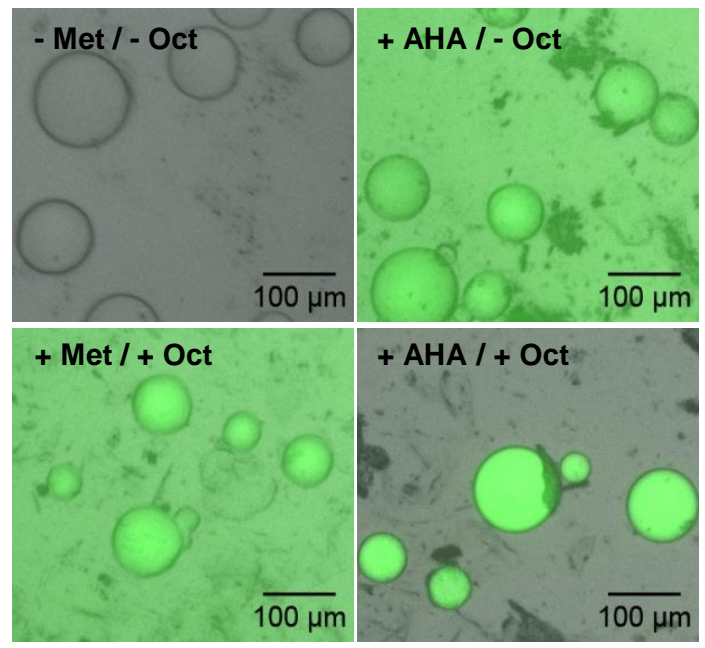

Figure 6. CFPS-i of sGFP incorporating non-natural amino acids by copper-free click chemistry. Fluorescence microscopy imaging overlaying brightfield and sGFP signals after the CFPS-i was completed. The images show the reaction in presence of each amino acid: Met (methionine) or AHA (L-azidohomoalanine) and the agarose beads activated or not with cyclooctyne (oct) groups.

Although the incorporation of non-natural amino acids for protein-based biomaterials manufacturing has been already described, ${ }^{38-40}$ here we have coupled the protein synthesis with a cyclooctyne/azide click-immobilization in one-pot concurrent process, unlike two-pot conventional methods where firstly azide- or alkyne-amino acids are incorporated either in vivo or in vitro into the protein. Then, the modified protein is purified and incubated with solid particles functionalized either with alkyne or azide groups. ${ }^{41,}{ }^{42}$ For a more precise site-selective immobilization, the CFPS-i developed here can be coupled to cell-free AMBER system, by using specific tRNAs to insert nonnatural amino acids in unique positions. ${ }^{43}$ Therefore, we demonstrate that the cooper-free clickimmobilization based on cyclooctyne/azide pairs is compatible with the protein synthesis according to the broad bio-orthogonality of this chemistry. ${ }^{44}$ 


\section{In vitro synthesis and selective co-immobilization of two proteins fused to different tags on the same carrier}

The co-immobilization of different enzymes on the same carrier is a frequent strategy to assemble complex biological machineries like multi-enzyme systems. ${ }^{45,46}$ In order to gain selectivity during the co-immobilization protocol, we have applied the toolbox of polypeptide-tags herein developed to simultaneously synthesize two different proteins and selectively co-immobilize them on the same carrier through two different immobilization chemistries. As a proof of concept, we in vitro synthesized Cys-sGFP and an alcohol dehydrogenase from Bacillus stearothermophilus tagged with 6 histidines at its N-terminus (His-ADHBs). In order to evaluate orthogonality of our toolbox for CFPS-i, we prepared a new agarose-based heterofunctional carrier activated with both cobalt-chelates and disulfide groups (Figure S13). This new surface theoretically allows the co-immobilization of Cys- and His-tagged proteins simultaneously through two different immobilization chemistries based on reversible covalent disulfide and reversible metal coordination bonds, respectively (Figure 7A). This concept was demonstrated by incubating the heterofunctional carrier with the corresponding DNA plasmids and the cell-free extract for the one-pot co-expression and co-immobilization of His-ADHBs and Cys-sGFP. As a control reaction, we also expressed only Cys-sGFP in presence of the heterofunctional carrier.

A
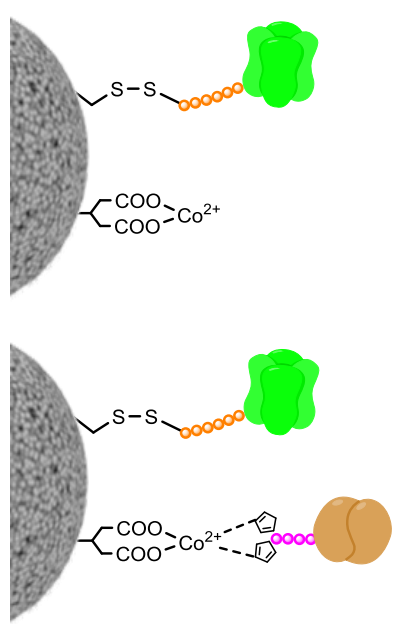

B
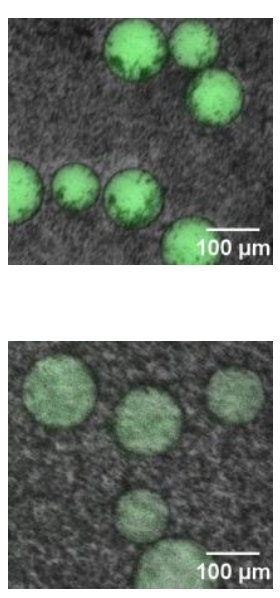

C

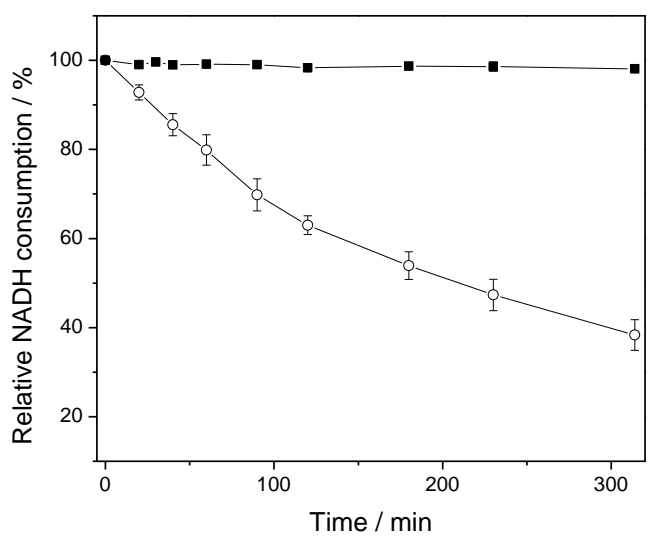

Figure 7. Co-expression and co-immobilization of two different proteins by two different chemistries. (A) Scheme of the immobilization of Cys-sGFP (up) and the co-immobilization of CyssGFP and His-ADHBs (down) on agarose beads activated with cobalt chelates and disulfide groups. 
(B) Overlay of brightfield and sGFP signals imaging. (C) Relative NADH consumption catalyzed by His-ADHBs and Cys-sGFP co-immobilized (white circles) and the control reaction with empty beads (black squares).

As read out to demonstrate the co-expression and co-immobilization of these two proteins, we visualized the fluorescence of Cys-sGFP by fluorescence microscopy (Figure 7B) and spectrophotometrically measured the activity of His-ADHBs attached to the carrier after washing steps to remove the protein synthesis machinery (Figure 7C). These experiments evidence that Cys-sGFP and His-ADHBs are co-expressed and co-immobilized on the same beads. However when we performed the CFPS-i using only the plasmid that encodes Cys-sGFP, the fluorescence intensity per bead was 4.22-times higher than when using the two different plasmids (Figure S14). This dissimilarity points out a possible competition between the two transcribed mRNA for the ribosomal machinery that may diminish the specific final yields for both Cys-sGFP and His-ADHBs. Karim and Jewett observed a similar decreasing effect on the protein expression levels when they tried the cell-free co-expression of several enzymes. ${ }^{47}$ According to Park et al., mRNA species must compete for a finite pool of ribosomes and aminoacyl-tRNAs when CFPS reaction mixtures are primed with several DNA templates, leading to unequal expression level of different proteins. Indeed, sGFP fluorescence was reported to be inversely proportional to translation rates of the co-expressed genes. ${ }^{48}$ Beside the fluorescence of the immobilized Cys-sGFP that reveals its proper folding and structurally innocuous immobilization, His-ADHBs in vitro synthesized and immobilized was catalytically active after CFPS-i (Figure 7C). The multimeric nature of this enzyme was not a hurdle for its successful expression and in situ immobilization by using the CFPS-i system, validating the feasibility of our technology for fabrication of solid platforms harboring more complex biological systems. Additionally, the orthogonality and selectivity of these two immobilization chemistries was demonstrated by selective elution of His-ADHBs in presence of EDTA, while the Cys-sGFP remained attached to the surface. Likewise, the Cys-sGFP was eluted to the solution by using thiolated compounds (Figure S15).

\section{Two-pot fabrication of immunoconjugates by CFPS-i}

The demonstrated plasticity of our methodology encouraged us to manufacture biomaterials for inmunosensing assays. In order to conceive a wide-ranging device, we decided to synthesize and immobilize protein A (ProA) by using CFPS-i. Materials functionalized with ProA mean a universal platform for binding to the $\mathrm{Fc}$ region of any Immunoglobulin $\mathrm{G}(\lg G) .{ }^{49}$ We utilized a previously 
reported plasmid that encodes ProA tagged with 6xHis-tag (His-ProA) at its $\mathrm{N}$-terminus under the control of the T7 promoter. ${ }^{50}$ In a first attempt, we incubated the plasmid DNA, the cell-free extract, the cobalt-activated beads and an $\lg G$ chemically conjugated with both rhodamine and alkaline phosphatase (AP-IgG-Rh) in one test tube to perform the CFPS-i of His-ProA and the subsequent IgG capture in one-pot. Unfortunately, the antibody was not able to be bound to the beads functionalized with the nascent ProA under the protein synthesis and immobilization conditions. In the light of this unsatisfactory result, we firstly expressed and immobilized His-ProA on cobalt-activated agarose beads in one pot (Figure 8A) and finally AP-IgG-Rh was incubated with those beads in a second pot (Figure 8B). The fluorescence and the enzyme activity conjugated to the $\lg G$ enabled to trace the antibody capture reaction. When AP-IgG-Rh was incubated with AG-Co lacking the His-ProA, we neither visualize fluorescence inside the beads nor detect enzyme activity after sample washing (Figure S16).

As a result, the fluorescence of the AP-lgG-Rh was uniformly distributed on the His-ProA agarose beads, pointing an optimal antibody density for antigen recognition as previously reported for the direct immobilization of IgG on porous agarose particles. ${ }^{16,51}$ However, the labeled antibody was not quantitatively captured by the beads functionalized with His-ProA; $43 \%$ of the offered antibody was immobilized (Figure S16). Hence, we demonstrate that His-ProA can be synthesized and immobilized in one-pot retaining its capacity to selectively capture conjugated IgGs, giving rise a two-step process for the fabrication of immobilized immunoconjugates. These results suggest that the protein synthesis conditions inhibit the affinity interaction between the Fc region of the antibody and the His-ProA. This strategy may contribute to developing innovative immunological platforms not only for manufacturing tailor-made biosensors and pull-down systems ${ }^{52}$ but also for immunotherapy. ${ }^{32}$ 
A
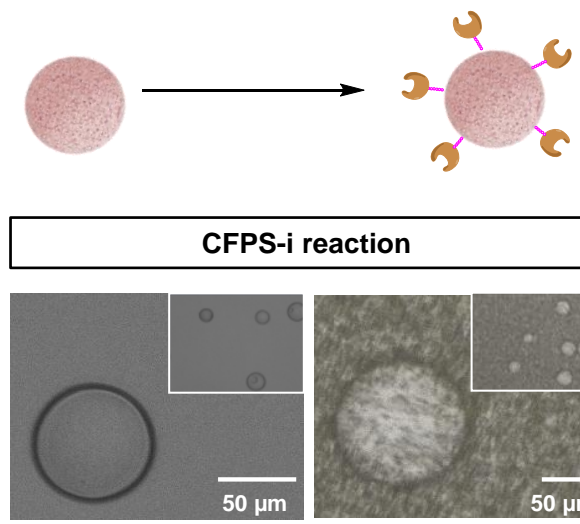

$t=0 h$
B

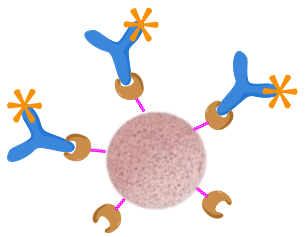

Incubation with IgG

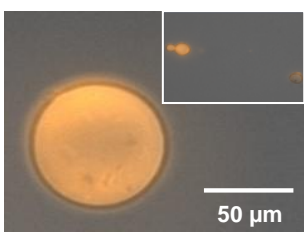

Figure 8. Two-pot synthesis of immunoconjugates by using CFPS-i. (A) Scheme and fluorescence microscopy imaging of CFPS-i of His-ProA (brown) on AG-Co (pink). (B) Scheme and fluorescence microscopy imaging of IgGs (blue) labeled with rhodamine (orange star) and captured by proteins A on AG-Co.

\section{CONCLUSION}

In summary, we have coordinated protein synthesis and immobilization in one-pot by developing a set of plasmids ready to use with suspensions of cell-free protein synthesis and solid materials to fabricate on-demand protein-based biomaterials. This technology is fully portable since it requires neither specialized equipments nor infrastructures. Here, we demonstrate that the in vitro synthesis of protein is orthogonal to a variety of solid materials as carriers and a diversity of immobilization chemistries that attach the nascent proteins to the carriers (see a summary in Table 1). We also exploited this system to produce multimeric proteins and biological conjugates (IgG-proteinA). However the optimal synthesis of larger proteins with quaternary structure and the in situ formation of biological complexes through specific protein-protein interactions will be some of the most important challenges this technology will must face in the near future.

The examples reported in this work encourage us to expand the CFPS-i technology to continuous-flow manufacturing of protein-based biomaterials as already proposed for the synthesis of active pharmaceutical ingredients ${ }^{53,54}$ and for bionanotechnology solutions. For instance, the integration of the CFPS-i platform into automated and miniaturized systems under GMP (good manufacture practices) conditions will undoubtedly boost the on-demand manufacturing of proteinbased biomaterials at the point-of-care, and the discovery of new biomaterials by prototyping their fabrication and shortening their processing time. In this sense, Schellekens et al. have reviewed some 
of the clues to make individualized drugs manufacturing a reality. ${ }^{55}$ They suggest that evolving from large scale pharmaceutical production to low scale and automated models, one can address the manufacturing of drugs for ultra-orphan and highly-rare diseases in a cost-efficient manner. Hence, we foresee our technology to play an important role in the personalized medicine when nanotechnology definitively bursts into clinical treatments.

Table 1. Description of synthesized biomaterials.

\begin{tabular}{|c|c|c|c|}
\hline Tagged protein & Binding material & Binding interaction & Elution reagent \\
\hline His-sGFP & $\begin{array}{c}\text { AG-Co } \\
\text { (Cobalt chelates activated agarose) }\end{array}$ & Metal coordination bond & Imidazol \\
\hline His-ADHBs & $\begin{array}{l}\text { Cobalt chelates and disulfide groups } \\
\text { activated agarose }\end{array}$ & Metal coordination bond & Imidazol \\
\hline His-ProA & $\begin{array}{c}\text { AG-Co } \\
\text { (Cobalt chelates activated agarose) } \\
\end{array}$ & Metal coordination bond & Imidazol \\
\hline \multirow{3}{*}{ Cys-sGFP } & $\mathrm{Pu}-\mathrm{E}$ & Covalent & - \\
\hline & $\mathrm{Au}-\mathrm{NRs}$ & Covalent & - \\
\hline & $\begin{array}{c}\text { Cobalt chelates and disulfide groups } \\
\text { activated agarose }\end{array}$ & Thiol exchange & DTT \\
\hline \multirow{2}{*}{ Lys-sGFP } & $\mathrm{SiO}_{2} \mathrm{NPs}$ & Ion exchange & Sodium chloride \\
\hline & Glass slide & Ion exchange & Sodium chloride \\
\hline $\begin{array}{l}\text { sGFP containing } \\
\text { AHA }\end{array}$ & Cyclooctyne activated agarose & $\begin{array}{l}\text { Copper-free click } \\
\text { chemistry }\end{array}$ & - \\
\hline
\end{tabular}

\section{METHODS}

Reagents and Materials: S30/T7 High yield protein expression system was supplied by Promega Corporation (Fitchburg, WI). Rhodamine B Isothiocyanate, Dibenzocyclooctyne-amine, Anti-Rabbit IgG (whole molecule)-Alkaline Phosphatase antibody produced in goat, 4-Nitrophenyl phosphate disodium salt hexahydrate (pNPP), 5,5'-Dithiobis(2-nitrobenzoic acid), 1,4-Dithiothreitol (DTT), Ethylenediaminetetraacetic acid (EDTA), Cyanogen bromide-activated Sepharose 4B, L-Lysine, LCysteine, L-Histidine and primers were acquired from Sigma-Aldrich (St. Louis, IL). Click-it AHA (Lazidohomoalanine) was supplied by Thermo Fisher Scientific. 6 channel $\mu$-Slide $\mathrm{VI}^{0.4}$ was purchased from ibidi (Planegg, Germany) and epoxy methacrylate beads Lifetech ECR8204 (Pu-E) were kindly donated by Purolite Ltd (Llantrisant, UK). The Colloidal nanofabrication platform from CICBiomaGUNE synthesized the AuNR and $\mathrm{SiO}_{2} \mathrm{NP}$ (See supporting information, section 7). Agarosebased materials monofunctionalized with cyclooctyne groups and heterofunctionalized with both 
disulfide and cobalt-chelates groups were fabricated using plain agarose beads purchased from ABT technologies (Madrid, Spain) (See supporting information, section 1).

Genetic engineering a toolbox of plasmids: Plasmid pET-28b(+) (Novagen) was used for cloning and protein expression. The genetic construction His-sGFP_pET28b was developed in a previous work. ${ }^{56}$ We designed eight primers (Table S1) for cloning Cys-sGFP, Lys-sGFP and untagged sGFP using as template the plasmid His-sGFP_pET28b. Firstly, we amplified by PCR the sequence upstream of the sGFP gene using primers 3 and 8 for the construction of Cys-sGFP_pET28b. Secondly, the downstream sequence was amplified with primers 7 and 4 . Finally, we performed an overlapping PCR by using primers 3 and 4 , and the previous PCR products as megaprimers. The pET-28b vector was digested with the restriction enzymes Ncol and Xhol. Afterwards, the overlapping PCR product and the digested vector were purified and ligated by homologous recombination in E.coli strain $\mathrm{DH} 10 \beta$ (laboratory stock). The same procedure was carried out for the genetic construction of Lys-sGFP_pET28b with primers 3 and $6 ; 5$ and $4 ; 3$ and 4 . Cloning of sGFP_pET28b was accomplished with primers 2 and 1 for the amplification of untagged-sGFP sequence. The resulting PCR product and the vector were further digested with $\mathrm{Ncol}$ and Xhol. After the dephosphorilation and purification of the DNA fragments, we ligated the DNA fragments and transformed them in BL21-Gold (DE3). All molecular biology protocols were performed using standard methods. ${ }^{57}$

In vitro cell-free protein synthesis and immobilization: The CFPS system was composed of $40 \%$ of S30 Premix Plus and $30 \%$ of T7/S30 extracts. In case of click reactions, we mixed $40 \%$ of S30 Premix without amino acids, $10 \%$ of amino acid mixture minus methionine, $10 \%$ of amino acid (methionine or L-AHA) and $30 \%$ of T7/S30 extracts. We added $1 \mu \mathrm{g}$ of DNA template and $1 \mathrm{mg}$ of carriers to the cell lysate-based expression system at final volume of $50 \mu \mathrm{L}$, unless otherwise specified. The reactions were performed at $37^{\circ} \mathrm{C}$ and $1200 \mathrm{rpm}$ for 12 hours in an Eppendorf Thermomixer R. 1mM CTAB was added to CFPS-i reactions with Pu-E and AuNR to avoid unspecific hydrophobic interactions and particle aggregation, respectively. Once the CFPS was finished, protein-based biomaterials were easily purified from the reaction mixture by rapid and low-cost filtration using mini Bio-spin chromatography columns.

Determination of His-sGFP loading on AG-Co: Solutions of in vivo expressed sGFP were incubated with AG-Co for 1 hour to achieve protein loadings from $0.5 \mathrm{mg} / \mathrm{g}$ to $5 \mathrm{mg} / \mathrm{g}$. The sGFP fluorescence from 10 beads of each loading was analyzed by ZEN 2012. The results were plotted in a calibration curve to estimate the CFPS-i of His-sGFP on AG-Co. 
Enzymatic activity assay: The enzymatic assays were carried out in a Varioskan ${ }^{\mathrm{TM}}$ Flash Multimode Reader (Thermo Scientific) using 96-well plates. For both soluble and immobilized enzyme the activity was measured under orbital shaking integrated into the reader. See supporting information section 2 for more details.

Fluorescence microscopy imaging: After the CFPS-i was completed, $10 \mu \mathrm{L}$ of the reaction mixture were placed on a channel of 6 channel $\mu$-Slide $\mathrm{VI}^{0.4}$. The brightfield transmission and fluorescence ( $\lambda_{\text {exc }}: 470 \mathrm{~nm}, \lambda_{\text {em }}: 500-550 \mathrm{~nm}$ ) images were obtained with a Cell Observer (Axio Observer, Zeiss) microscope. In order to better visualize AuNR and $\mathrm{SiO}_{2} \mathrm{NP}$, the samples were 3 times washed with 25 $\mathrm{mM}$ sodium phosphate buffer ( $\mathrm{pH}$ 7) before their visualization. Images were processed with ZEN 2012 (Zeiss) and FIJI/ImageJ softwares.

\section{ABBREVIATIONS}

$\begin{array}{ll}\text { AG-Co } & \text { Agarose microbeads activated with Cobalt chelates groups } \\ \text { AP-IgG-Rh } & \text { Inmunoglobulin G chemically conjugated with both rhodamine and alkaline phosphatase } \\ \text { AuNRs } & \text { Gold nanorods } \\ \text { CFPS } & \text { Cell-free protein synthesis } \\ \text { CFPS-i } & \text { Cell-free protein synthesis and immobilization } \\ \text { Cys-sGFP } & \text { sGFP tagged with poly-(6x)Cys } \\ \text { His-ADHBs } & \text { Alcohol dehydrogenase from Bacillus stearothermophilus tagged with poly-(6X)His } \\ \text { His-ProA } & \text { Protein A tagged with poly-(6X)His } \\ \text { His-sGFP } & \text { sGFP tagged with poly-(6X)His } \\ \text { L-AHA } & \text { L-azidohomoalanine } \\ \text { Lys-sGFP } & \text { sGFP tagged with poly-(6x)Lys } \\ \text { Pu-E } & \text { Methacrylate microbeads activated with epoxide groups } \\ \text { sGFP } & \text { superfolded Green Fluorescent Protein } \\ \text { SiO }{ }_{2} N P s & \text { Silica nanoparticles }\end{array}$

\section{AUTHOR INFORMATION}

Author contribution: A.I.B.M. and F.L.G. designed research; A.I.B.M., I.L., A.S.I., and F.L.G. performed research; A.I.B.M. and F.L.G. analyzed data, wrote and edited the manuscript.

Conflict of interest: The authors declare no conflict of interest.

\section{SUPPORTING INFORMATION}

Table S1 and figures S1-S16. 


\section{ACKNOWLEDGEMENTS}

We want to acknowledge COST action CM103-System biocatalysis, IKERBASQUE, Basque foundation of Science and ARAID, Aragon I+D foundation for funding to Dr. F. López-Gallego and to Spanish Minister MINECO (BIO2014-61838-EXP) for funding A. I. Benítez-Mateos. We want to thank Dr. C. Araya-Callís and Dr. R. P. Richter for the kind donation of plasmid encoding His-ProA protein. Likewise, we also thank Dr. A. Basso from Purolite Ltd for kindly donating us some samples of Purolite $\AA^{\circledR}$ resins.

\section{REFERENCES}

1. Nagamune, T. (2017) Biomolecular engineering for nanobio/bionanotechnology. Nano Converg. $4,9$.

2. Li, L., He, Z. Y., Wei, X. W., Wei, Y.Q. (2016) Recent advances of biomaterials in biotherapy. Regen. Biomater. 3, 99-105.

3. Wu, P., Castner, D. G., Grainger, D. W. (2008) Diagnostic devices as biomaterials: a review of nucleic acid and protein microarray surface performance issues. J. Anal. Bioanal. Tech.19, 725-753.

4. Prasad, A., Mahato, K., Maurya, P. K., Chandra, P. (2016) Biomaterials for Biosensing Applications. J. Anal. Bioanal. Tech. 7, e124.

5. Karfeld-Sulzer, L. S., Waters, E. A., Kohlmeir, E. K., Kissler, H., Zhang, X., Kaufman, D. B., et al. (2011) Protein Polymer MRI Contrast Agents: Longitudinal Analysis of Biomaterials in Vivo. Magn. Reson. Med. 65, 220-228.

6. Gagner, J.E., Kim, W., Chaikof, E. L. (2014) Designing Protein-Based Biomaterials for Medical Applications. Acta Biomater. 10, 1542-1557.

7. Wei, Q., Becherer, T., Angioletti-Uberti, S., Dzubiella, J., Wischke, C., Neffe, A.T., et al. (2014) Protein Interactions with Polymer Coatings and Biomaterials. Angew. Chem. Int. Ed. 53, 8004-8031.

8. Editorial. (2017) Patient-centered drug manufacture. Nat. Biotechnol. 35, 485.

9. Rosenblum, G., Cooperman, B. S., (2014) Engine out of the chassis: Cell-free protein synthesis and its uses. FEBS Lett. 588, 261-268.

10. Caschera, F., Noireaux, V., (2014) Synthesis of $2.3 \mathrm{mg} / \mathrm{ml}$ of protein with an all Escherichia coli cell-free transcription-translation system. Biochim. 99, 162-168. 
11. Li, J., Lawton, T. J., Kostecki, J. S., Nisthal, A., Fang, J., Mayo, S. L., et al. (2016) Cell-free protein synthesis enables high yielding synthesis of an active multicopper oxidase. Biotechnol. J. 11, 212-218.

12. Pardee, K., Slomovic, S., Nguyen, P. Q., Lee, J. W., Donghia, N., Burrill, D., et al. (2016) Portable, On-Demand Biomolecular Manufacturing. Cell. 167, 248-259.

13. Smith, M. T., Berkheimer, S. D., Werner, C. J., Bundy, B. C. (2014) Lyophilized Escherichia coli-based cell-free systems for robust, high-density, long-term storage. Biotechniques. 56, 186-193.

14. Karig, D. K., Bessling, S., Thielen, S., Wolfe, J. (2017) Preservation of protein expression systems at elevated temperatures for portable therapeutic production. J. R. Soc. Interface. 129, pii: 20161039.

15. Bhide, M., Natarajan, S., Hresko, S., Aguilar, C., Bencurova, E. (2014) Rapid in vitro protein synthesis pipeline: a promising tool for cost-effective protein array design. Mol. Biosyst. 10, 1236-1245.

16. Batalla, P., Bolívar, J.M., Lopez-Gallego, F., Guisan, J.M. (2012) Oriented covalent immobilization of antibodies onto heterofunctional agarose supports: A highly efficient immuno-affinity chromatography platform. J. Chromatogr. A. 1262, 56-63.

17. Catherine, C., Lee, S. W., Ju, J. W., Kim, H. C., Shin, H. I., Kim, Y. J., et al. (2015) Cell-Free Expression and In Situ Immobilization of Parasite Proteins from Clonorchis sinensis for Rapid Identification of Antigenic Candidates. PloS One. 10, e0143597.

18. Hall, S. R., Hnilova, M., Grosh, C., Fong, H., Baneyx, F., Schwartz, D., et al. (2012) Engineered Escherichia coli Silver-Binding Periplasmic Protein That Promotes Silver Tolerance. Appl. Environ. Microbiol. 78, 2289-2296.

19. Yang, M., Choi, B. G., Park, T. J., Heo, N. S., Hong, W.H., Lee, S. Y. (2011) Site-specific immobilization of gold binding polypeptide on gold nanoparticle-coated graphene sheet for biosensor application. Nanoscale. 3, 2950-2956.

20. Bodelón, G., Mourdikoudis, S., Yate, L., Pastoriza-Santos, I., Pérez-Juste, J., Liz-Marzán, L. M. (2014) Nickel Nanoparticle-Doped Paper as a Bioactive Scaffold for Targeted and Robust Immobilization of Functional Proteins. ACS Nano. 8, 6221-6231.

21. Schwaminger, S. P., Blank-Shim, S. A., Scheifele, I., Fraga-Garcia, P., Berensmeier, S. (2017) Peptide binding to metal oxide nanoparticles. Faraday Discuss. 204, 233-250. 
22. Wiesbauer, J., Bolivar, J. M., Mueller, M., Schiller, M., Nidetzky, B. (2011) Oriented Immobilization of Enzymes Made Fit for Applied Biocatalysis: Non-Covalent Attachment to Anionic Supports using Zbasic2 Module. ChemCatChem. 3, 1299-303.

23. López-Gallego, F., Acebrón, I., Mancheño, J. M., Raja, S., Lillo, M. P., Guisán, J. M. (2012) Directed, Strong, and Reversible Immobilization of Proteins Tagged with a $\beta$-Trefoil Lectin Domain: A Simple Method to Immobilize Biomolecules on Plain Agarose Matrixes. Bioconjug. Chem. 23, 565-573. 24. Yunker, P. J., Asahara, H., Hung, K. C., Landry, C., Arriaga, L. R., Akartuna, I., et al. (2016) One-pot system for synthesis, assembly, and display of functional single-span membrane proteins on oil-water interfaces. Proc. Natl. Acad. Sci. U. S. A. 113, 608-613.

25. Ramachandran, N., Raphael, J. V., Hainsworth, E., Demirkan, G., Fuentes, M. G., Rolfs, A., et al. (2008) Next generation high density self assembling functional protein arrays. Nat. Methods. 5, 535-538.

26. He, M., Taussig, M. J. (2001) Single step generation of protein arrays from DNA by cellfree expression and in situ immobilisation (PISA method). Nucleic Acids Res. 29, E73-3.

27. He, M., Liu, H., Turner, M., Taussig, M. J. (2009) Detection of protein-protein interactions by ribosome display and protein in situ immobilisation. N. Biotechnol. 26, 277-281.

28. He, M., Stoevesandt, O., Taussig, M. J. (2007) In situ synthesis of protein arrays. Curr. Opin. Biotechnol. 19, 4-9

29. Lee, K. H., Kwon, Y. C., Yoo, S. J., Kim, D. M. (2010) Ribosomal synthesis and in situ isolation of peptide molecules in a cell-free translation system. Protein Expr. Purif. 71, 16-20.

30. Lee, K. H., Lee, K. Y., Byun, J. Y., Kim, B. G., Kim, D. M. (2012) On-bead expression of recombinant proteins in an agarose gel matrix coated on a glass slide. Lab Chip. 12, 1605-1610.

31. Byun, J. Y., Lee, K. H., Lee, K. Y., Kim, M. G., Kim, D. M. (2013) In-gel expression and in situ immobilization of proteins for generation of three dimensional protein arrays in a hydrogel matrix. Lab Chip. 13, 886-891.

32. Wang, C., Ye, Y., Hu, Q., Bellotti, A., Gu, Z. (2017) Tailoring Biomaterials for Cancer Immunotherapy: Emerging Trends and Future Outlook. Adv. Mater. 29, 1606036.

33. Singh, M. I., Jain, V. (2013) Tagging the Expressed Protein with 6 Histidines: Rapid Cloning of an Amplicon with Three Options. PloS One. 8. 
34. Kwon, Y. C., Jewett, M.C. (2015) High-throughput preparation methods of crude extract for robust cell-free protein synthesis. Sci. Rep. 5, 8663.

35. Tuckey, C., Asahara, H., Zhou, Y., Chong, S. (2014) Protein Synthesis Using A Reconstituted Cell-Free System. Curr. Protoc. Mol. Biol. 108, 16.31.1-22.

36. Frasconi, M., Mazzei, F., Ferri, T. (2010) Protein immobilization at gold-thiol surfaces and potential for biosensing. Anal. Bioanal. Chem. 398, 1545-1564.

37. Hall, D. A., Ptacek, J., Snyder, M. (2007) Protein Microarray Technology. Mech. Ageing Dev. $128,161-167$.

38. Albayrak, C., Swartz, J.R. (2013) Cell-free co-production of an orthogonal transfer RNA activates efficient site-specific non-natural amino acid incorporation. Nucleic Acids Res. 41, 59495963.

39. Hatzenpichler, R., Scheller, S., Tavormina, P. L., Babin, B. M., Tirrell, D. A., Orphan, V. J. (2014) In situ visualization of newly synthesized proteins in environmental microbes using amino acid tagging and click chemistry. Environm. Microbiol. 16, 2568-2590.

40. Wu, J. C., Hutchings, C. H., Lindsay, M. J., Werner, C. J., Bundy, B. C. (2015) Enhanced enzyme stability through site-directed covalent immobilization. J. Biotechnol. 193, 83-90.

41. Smith, M. T., Wu, J. C., Varner, C. T., Bundy, B. C. (2013) Enhanced protein stability through minimally invasive, direct, covalent, and site-specific immobilization. Biotechnol. Prog. 29, 247-254.

42. Bundy, B. C., Swartz, J.R. (2010) Site-Specific Incorporation of p-Propargyloxyphenylalanine in a Cell-Free Environment for Direct Protein-Protein Click Conjugation. Bioconjug. Chem. 21, 255263.

43. Drabkin, H.J., RajBhandary, U. L. (1998) Initiation of Protein Synthesis in Mammalian Cells with Codons Other Than AUG and Amino Acids Other Than Methionine. Mol. Cell Biol. 18, 5140-5147. 44. Raliski, B. K., Howard, C.A., Young, D. D. (2014) Site-Specific Protein Immobilization Using Unnatural Amino Acids. Bioconjug. Chem. 25,1916-1920.

45. Burdick, B. A., Schaeffer, J.R. (1987) Co-immobilized coupled enzyme systems on nylon mesh capable of gluconic and pyruvic acid production. Biotechnol. Lett. 9, 253-258.

46. Mateo, C., Grazu, V., Palomo, J. M., Lopez-Gallego, F., Fernandez-Lafuente, R., Guisan, J. M. (2007) Immobilization of enzymes on heterofunctional epoxy supports. Nat. Protoc. 2, 1022-1033. 
47. Karim, A. S., Jewett, M. C. (2016) A cell-free framework for rapid biosynthetic pathway prototyping and enzyme discovery. Metab. Eng. 36, 116-126.

48. Park, Y. J., Lee, K. H., Kim, D. M. (2017) Assessing translational efficiency by a reporter protein co-expressed in a cell-free synthesis system. Anal. Biochem. 518, 139-142.

49. Tarrant, R. D. R., Velez-Suberbie, M. L., Tait, A. S., Smales, C. M., Bracewell, D. G. (2012) Host cell protein adsorption characteristics during protein a chromatography. Biotechnol. Prog. 28, 1037-1044.

50. Dubacheva, G. V., Araya-Callis, C., Geert, V. A., Fairhead, M., Codée, J., Howarth, M., et al. (2017) Controlling Multivalent Binding through Surface Chemistry: Model Study on Streptavidin. J. Am. Chem. Soc. $139,4157-4167$.

51. Ferrari, M., Barreto, R., Jackson, E., Guisan, J.M., Lopez-Gallego, F., Betancor, L. (2015) Optimizing the biological activity of Fab fragments by controlling their molecular orientation and spatial distribution across porous hydrogels. Process Biochem. 50, 1565-1571.

52. Brymora, A., Valova, V. A., Robinson, P. J. (2001) Protein-Protein Interactions Identified by Pull-Down Experiments and Mass Spectrometry. Curr. Protoc. Cell Biol. 17, 17.5.

53. Adamo, A., Beingessner, R. L., Behnam, M., Chen, J., Jamison, T. F., Jensen, K. F., et al. (2016) On-demand continuous-flow production of pharmaceuticals in a compact, reconfigurable system. Science. 352, 61.

54. Timm, A. C., Shankles, P. G., Foster, C. M., Doktycz, M, J., Retterer, S. T. (2016) Toward Microfluidic Reactors for Cell-Free Protein Synthesis at the Point-of-Care. Small. 12, 810-817.

55. Schellekens, H., Aldosari, M., Talsma, H., Mastrobattista, E. (2017) Making individualized drugs a reality. Nat. Biotechnol. 35, 507-513.

56. Bolivar, J. M., Hidalgo, A., Sánchez-Ruiloba, L., Berenguer, J., Guisán, J. M., López-Gallego, F. (2011) Modulation of the distribution of small proteins within porous matrixes by smart-control of the immobilization rate. J. Biotechnol. 155, 412-420.

57. Denman, A. M. (1983) Molecular Cloning: a Laboratory Manual. Immunology. 49, 411. 